\title{
Transport d'oxygène chez le malade grave
}

La relation entre la consommation d'oxygène $\left(\dot{\mathrm{VO}}_{2}\right)$ et le transport en oxygène $\left(\mathrm{DO}_{2}\right)$ fait l'objet d'un intérêt croissant en soins intensifs. $\mathrm{La} \mathrm{DO}_{2}$ est le produit du débit cardiaque par le contenu artériel en oxygène $\left(\mathrm{CaO}_{2}\right)$. Le $\mathrm{CaO}_{2}$ est à son tour déterminé par la concentration en hémoglobine et la saturation de celle-ci. Un taux d'hémoglobine bas doit être corrigé par une transfusion lorsqu'il met la $\mathrm{DO}_{2}$ en danger. De même une pression partielle artérielle en oxygène $\left(\mathrm{PaO}_{2}\right)$ abaissée doit être corrigée chez le patient hypoxémique : l'interprétation d'une valeur de débit cardiaque est plus compliquée puisque le débit cardiaque doit s'adapter aux besoins en oxygène de l'organisme. Un débit cardiaque bas doit être traité seulement lorsque la demande en oxygène est normale ou élevée : par contre, il peut être encore adéquat chez des patients anesthésiés, hypothermes, ventilés artificiellement. A l'autre extrême, puisque la demande en oxygène des tissus est généralement augmentée dans les affections aiguës associées au sepsis, à la chirurgie, au traumatisme ou à d'autres états inflammatoires, le débit cardiaque doit parfois s'accroître de manière importante dans ces circonstances. Jusqu'à un certain point, on peut comparer ces conditions à l'exercice musculaire.

Lorsque la demande en oxygène augmente de manière aiguë, la $\mathrm{DO}_{2}$ doit également s'élever sans délai. Une augmentation de la $\mathrm{PaO}_{2}$ au-dessus de la normale n'augmente pas beaucoup la $\mathrm{DO}_{2}$ en raison de la saturation presque complète de l'hémoglobine. De même une augmentation du taux d'hémoglobine au dessus de la normale ne peut être obtenue rapidement et n'aurait qu'une efficacité très mitigée sur la disponibilité en oxygène de la cellule en raison de l'augmentation simultanée de la viscosité sanguine. Dès lors, le débit cardiaque et la distribution du flux sanguin dans l'organisme représentent les seuls facteurs qui peuvent immédiatement et efficacement augmenter la disponibilité en oxygène des cellules. Ainsi, un débit cardiaque consideré comme normal peut être encore inadéquat ou insuffisant lorsque la demande en oxygène des tissus est augmentée.

L'étude de la relation entre $\mathrm{VO}_{2}, \mathrm{DO}_{2}$ et demande en oxygène aide à interpréter les valeurs de débit cardiaque. Elle permet également le développement d'un concept unique définissant tous les types d'insuffisance circulatoire aiguë et peut indiquer des options thérapeutiques susceptibles d'améliorer la fonction cellulaire et de prévenir la défaillance multisystémique et le décès.

\section{La relation $\mathrm{VO}_{2} / \mathrm{DO}_{2}$ dans les conditions physiologiques}

Dans les conditions stables physiologiques, la $\dot{\mathrm{VO}} \mathrm{O}_{2}$ est égale à la demande en oxygène. En l'absence d'hypoxie cellulaire, les taux de lactate sanguin sont normaux aux environs de $1 \mathrm{mmol} \cdot \mathrm{L}^{-1}$. Si la $\mathrm{DO}_{2}$ diminue en présence d'hypoxémie, d'anémie ou d'un bas débit cardiaque, la $\mathrm{VO}_{2}$ reste relativement stable pour un large spectre de valeurs de $\mathrm{DO}_{2}$. En effet, l'extraction d'oxygène, c'està-dire le rapport de la différence artério-veineuse sur la $\mathrm{CaO}_{2}$, ou le rapport $\mathrm{VO}_{2} / \mathrm{DO}_{2}$, augmente. Le principal mécanisme sous-jacent est situé dans la microvasculature où le réseau capillaire se dilate pour apporter davantage d'oxygène aux cellules. $\mathrm{La} \mathrm{V}_{2}$ est dès lors indépendante de la $\mathrm{DO}_{2}$. De toute évidence, ceci n'est vrai que lorsque la demande en oxygène est stable. Si la demande en oxygène devait s'accroitre pour n'importe quelle raison (au cours de l'exercice, par exemple), le débit cardiaque et donc la $\mathrm{DO}_{2}$ devraient immédiatement augmenter.

Lorsque la $\mathrm{DO}_{2}$ tombe en-dessous d'une valeur critique, l'augmentation de l'extraction d'oxygène devient moins efficace si bien que la $\mathrm{V}_{2} \mathrm{~s}$ 'abaisse pour devenir alors dépendante de la $\mathrm{DO}_{2}$. Chez l'animal intact, ce niveau critique est aux environs de 8 à $10 \mathrm{ml} \cdot \mathrm{kg}^{-1}$. $\min ^{-1} \cdot{ }^{1-3}$ Comme montré par Cain ${ }^{1}$ il y a plus de 25 ans, ces états sont associés à une hypoxie tissulaire que reflète une augmentation des taux sanguins de lactate. Chez l'agneau conscient, Fahey et Lister ${ }^{2}$ ont observé que la $\dot{\mathrm{V}} \mathrm{O}_{2}$ devenait dépendante de la $\mathrm{DO}_{2}$ lorsque le débit cardiaque était réduit en-dessous d'un seuil critique. L'acidose lactique est également apparue lorsque ce niveau critique de débit cardiaque était atteint. Donc, la dépendance de la $\mathrm{VO}_{2}$ par rapport à la $\mathrm{DO}_{2}$ caractérise les types de choc circulatoire associés à une réduction importante du débit cardiaque : les formes de choc hypovolémique, cardiogénique et obstructif. Dans les formes pures de choc cardiogénique ou obstructif, les capacités d'extraction d'oxygène de l'organisme sont toujours très efficaces si bien que les taux de lactate commencent à augmenter dans les situations extrèmes qui sont associées à une mortalité élevée. Dans le choc hypovolémique, une combinaison d'un débit cardiaque

Département de Soins Intensifs, Hôpital Universitaire Erasme, Université Libre de Bruxelles, Belgiquc. 
abaissé (liée à une réduction du retour veineux) et une diminution du volume sanguin rendent compte de l'hypoxie tissulaire.

\section{La dépendance de la $\mathrm{V}_{2}$ par rapport a la $\mathrm{DO}_{2}$ au cours du sepsis}

Chez les patients présentant une infection sévère ou le syndrome de détresse respiratoire aiguë de l'adulte (SDRA), la $\mathrm{VO}_{2}$ peut rester dépendante de la $\mathrm{DO}_{2}$ même lorsque celle-ci est normale ou élevée. ${ }^{4,5}$ Ces états sont caractérisés par une demande en oxygène élevée si bien que le débit cardiaque et la $\mathrm{DO}_{2}$ devraient l'être également. Au même moment, l'extraction d'oxygène par les tissus est altérée au cours de ces états inflammatoires par plusieurs facteurs tels que le développement de microthromboses, la libération de nombreux médiateurs vasoactifs et l'altération de la fonction cellulaire endothéliale. ${ }^{6}$ Les altérations cellulaires du sepsis semblent apparaître plus tardivement. ${ }^{7}$

Plusieurs éléments indiquent que la dépendance pathologique de la $\dot{\mathrm{VO}}_{2}$ par rapport à la $\mathrm{DO}_{2}$, tout comme la dépendance physiologique, est associée à une hypoxie tissulaire et au développement de métabolisme anaérobique. Toutefois, les altérations d'extraction d'oxygène rendent l'organisme plus susceptible à une $\mathrm{DO}_{2}$ qui serait inappropriée. Sur le plan expérimental, Nelson et al. ${ }^{3}$ ont observé au cours de l'hémorragie progressive chez le chien que le niveau critique de $\mathrm{DO}_{2}$ en-dessous duquel apparait la dépendance de la $\mathrm{VO}_{2}$ par rapport à la $\mathrm{DO}_{2}$ était associé à l'apparition d'une augmentation des taux de lactate. Ces observations étaient en accord avec les études de Cain' dans lesquelles la $\mathrm{DO}_{2}$ était progressivement diminuée par une anémie progressive ou une hypoxémie. Quand un protocole similaire était appliqué après l'administration d'endotoxine ou de bactéries vivantes à l'animal, la même évolution était observée sauf que le niveau de $\mathrm{DO}_{2}$ critique était déplacé vers des valeurs plus élevées. ${ }^{3}$ Dès lors, une réduction de $\mathrm{DO}_{2}$ à un niveau toujours bien toléré chez l'animal intact pouvait être associée à une hypoxie tissulaire et une acidose lactique chez les animaux septiques.

Chez les patients aussi, la dépendance de la $\mathrm{VO}_{2}$ par rapport à la $\mathrm{DO}_{2}$ est généralement associée à une acidose lactique. Chez les patients présentant un sepsis et une acidose lactique, plusieurs investigateurs ${ }^{8-11}$ ont observé une augmentation de la $\dot{\mathrm{V}}_{2}$ associée à une augmentation du débit cardiaque après administration de liquides intraveineux. Une telle augmentation n'était pas observée chez les patients sans acidose lactique..$^{8-10}$ Fenwick $e t$ al. ${ }^{10}$ ont observé chez les patients avec SDRA que la transfusion sanguine augmentait la $\mathrm{VO}_{2}$ seulement chez ceux qui avaient des taux de lactate élevés. Par contre,
Annat et al. ${ }^{12} \mathrm{n}$ 'ont pas observé de dépendance de la $\dot{\mathrm{VO}}_{2}$ par rapport à la $\mathrm{DO}_{2}$ chez des patients ayant des taux de lactate normaux.

Nous avons étudié ${ }^{13}$ les réponses en $\mathrm{DO}_{2}$ et $\mathrm{VO}_{2}$ à l'administration d'une dose standard de $5 \mu \mathrm{g} \cdot \mathrm{kg}^{-1}$. $\min ^{-1}$ de dobutamine chez 78 malades graves avec insuffisance cardiaque ou sepsis. Dans les deux sousgroupes de patients, la $\dot{\mathrm{VO}}_{2}$ a augmenté seulement chez ceux qui avaient des taux élevés de lactate sanguin. Parmi les patients en SDRA du groupe septique, la $\dot{\mathrm{VO}}_{2}$ augmentait seulement lorsque l'acidose lactique était présente. $^{13}$

Les résultats de ces études et d'autres ${ }^{14}$ démontrent que le phénomène de dépendance de la $\dot{\mathrm{VO}}_{2}$ par rapport à la $\mathrm{DO}_{2}$ est observé seulement en présence d'hypoxie tissulaire comme l'indiquent les taux de lactate élevés. Donc l'augmentation de la $\dot{\mathrm{VO}}_{2}$ atteinte dans ces conditions est probablement bénéfique. Ceci est important parce qu'une autre interprétation serait que la quantité supplémentaire d'oxygène consommé l'est principalement par des systèmes qui ne produisent pas d'ATP au cours de la réaction inflammatoire. Cette utilisation de l'oxygène pourrait contribuer à la formation de radicaux libres d'oxygène et pourrait donc être délétère pour l'organisme. Si ces mécanismes devaient prendre place, ils seraient aussi observés en l'absence d'hypoxie tissulaire et d'acidose lactique. En présence d'hypoxie tissulaire toutefois l'oxygène additionnel est utilisé préférentiellement dans la mitochondrie puisque l'affinité des systèmes oxidatifsextramitochondriaux est moindre que celle de la chaîne de transport d'oxygène intramitochondriale (cytochrome aa3). L'utilisation d'oxygène pourrait prendre place également en dehors de la mitochondrie mais dans des stages de sepsis plus avancés lorsque la défaillance multi-organique est déjà présente.

$\mathrm{La} \mathrm{DO}_{2}$ insuffisante en présence de certaines affections aiguës caractérisées par une demande en oxygène accrue et une altération d'extraction d'oxygène peut être expliquée par la dépression myocardique qui est associée au sepsis sévère. La diminution de contractilité myocardique peut être documentée même lorsque le débit cardiaque est normal ou élevé. ${ }^{15-17}$

Ces éléments peuvent rendre compte des anomalies associées à toutes les formes d'insuffisance circulatoire aiguë (choc). Comme représenté à la Figure, les types de choc hypovolémique, cardiogénique et obstructif sont caractérisés par une réduction de la $\mathrm{DO}_{2}$ mais la demande en oxygène est relativement normale et l'extraction d'oxygène est bien préservée. D'autre part, le choc septique est caractérisé par une augmentation de la demande en oxygène, une altération de l'extraction d'oxygène et une limitation du débit par la dépression 


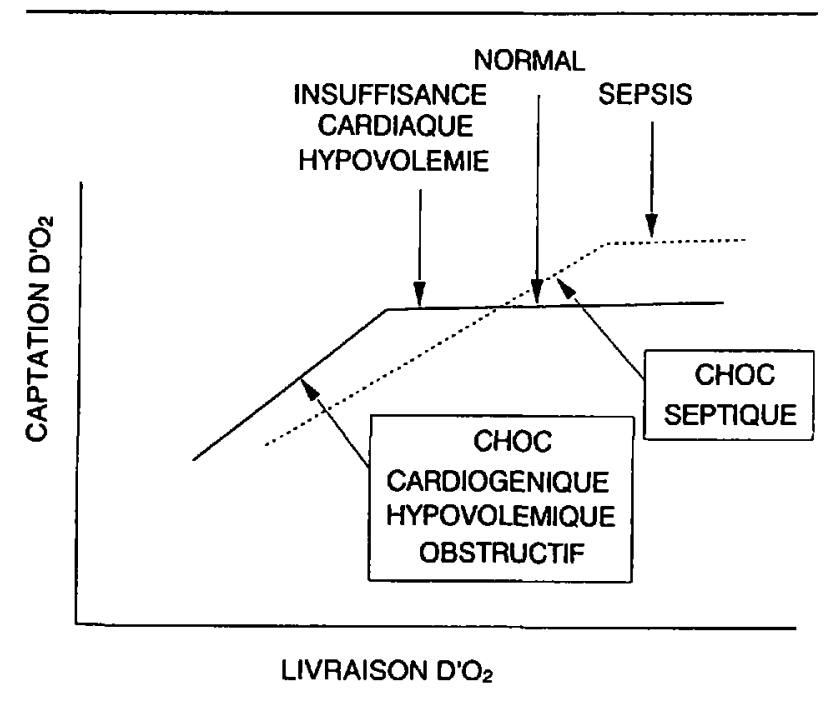

FIGURE Relation entre captation et livraison de l'oxygène chez le patient normal, dans l'insuffisance cardiaque, l'hypoxémie, la septicémie et le choc.

myocardique sous-jacente (parfois non suspectée). Le degré de dépression myocardique semble directement lié à la sévérité du sepsis et à la probabilité de décès. ${ }^{16,17}$

\section{Interprétation d'une valeur de débit cardiaque}

Au vu de ces observations, nous suggérons cinq étapes successives à l'interprétation du débit cardiaque (Tableau I).

La première étape est de ramener le débit cardiaque à la corpulence de l'individu. La référence à la surface corporelle permet de calculer l'index cardiaque.

La seconde étape est de déterminer la présence d'anémie ou d'hypoxémie et si la $\mathrm{DO}_{2}$ peut être en danger, ces éléments devraient être corrigés. Un niveau d'hémoglobine de $10 \mathrm{a} 11,5 \mathrm{~g} \cdot \mathrm{dl}^{-1}$ (ou un hématocrite de 30 à $35 \%$ ) est généralement recommandé. Une $\mathrm{PaO}_{2}$ d'au moins 60 $\mathrm{mmHg}$ doit être maintenue pour obtenir une saturation d'hémoglobine d'au moins $90 \%$.

La troisième étape consiste en la mesure simultanée de la saturation de l'hémoglobine du sang veineux mêlé $\left(\mathrm{S}_{\overline{\mathrm{O}}} \mathrm{O}_{2}\right)$. Les changements de $\mathrm{S}_{\bar{v}} \mathrm{O}_{2}$ accompagnent les changements du rapport $\dot{\mathrm{V}} \mathrm{O}_{2} / \mathrm{DO}_{2}$ : pour une $\dot{\mathrm{VO}}_{2}$

TABLEAU I Les 5 étapes dans l'interprétation du débit cardiaque chez un patient en etat critique :

I S'en référer à la surface corporelle (calcul de l'index cardiaque)

2 Elimincr hypoxémie ou anémic (et les corriger si présents)

3 Déterminer la $\mathrm{Sv}_{2}$

4 Déterminer un taux de lactate sanguin

5 "Challenger" la $\mathrm{VO}_{2}$ donnée, une diminution du débit cardiaque est associée à une diminution simultanée de la $\mathrm{Sv}_{2}$. L'interprétation de la $\mathrm{Sv}_{\mathbf{v}} \mathrm{O}_{2}$ est toutefois limitée par la possibilité d'altération des capacités d'extraction d'oxygène par les tissus. Le point d'extraction critique peut être défini dans des investigations précises de laboratoire. mais pas sur le plan clinique.

La quatrième étape consiste en la mesure d'une concentration en lactate sanguin. $\mathrm{La} \mathrm{Sv}_{2}$ ne peut se substituer au taux de lactate et vice versa parce que les deux ne sont pas bien corrélés excepté peut-être en présence d'une insuffisance cardiaque isolée. ${ }^{18}$ Les taux de lactate reflètent le degré de métabolisme anaérobie. Leur interprétation peut être compliquée par le fait qu'ils peuvent également augmenter dans d'autres conditions telles que les crises convulsives, le diabète décompensé ou certaines intoxications. D'une importance plus grande est le fait que les taux de lactate sanguin sont influencés non seulement par la prodution de lactate mais aussi par son élimination. Comme le lactate est métabolisé principalement par le foie, il peut rester élevé en particulier lorsque la fonction hépatique est altérée. Quoiqu'il en soit, les taux de lactate sont normaux chez les patients stables avec une insuffisance hépatique avancée si bien qu'une lactatémie normale reste un argument solide pour exclure la présence d'hypoxie tissulaire. ${ }^{19}$ Cette diminution de clearance du lactate peut compliquer l'interprétation de ces taux sanguins au cours de l'évolution d'un épisode d'insuffisance circulatoire aiguë. Dès lors, il est important de mesurer les taux de lactate de manière répétée pour permettre une interprétation dynamique de ces valeurs. Dans les meilleures circonstances associées à une bonne réponse à l'administration de liquides intraveineux, les taux de lactate diminuent d'au moins $10 \%$ au cours de la première heure de traitement. ${ }^{20}$ Le Tableau II montre comment le débit cardiaque est interprété en fonction de la $\mathrm{SviO}_{2}$ et des taux de lactate.

La cinquième étape consiste en une évaluation dynamique de la $\mathrm{VO}_{2}$ au cours d'une augmentation transitoire du débit cardiaque. $\mathrm{Ce}$ «challenge de la $\mathrm{VO}_{2}$ " peut être accompli par l'administration de liquides ou d'une transfusion sanguine. Bihari et al. ${ }^{4}$ ont montré qu'une dose de $5 \mathrm{ng} \cdot \mathrm{kg}^{-1} \cdot \min ^{-1}$ de prostacycline pouvait révéler la dépendance de la $\dot{\mathrm{VO}}_{2}$ par rapport à la $\mathrm{DO}_{2}$ chez des patients avec sepsis ou insuffisance respiratoire aiguë ; ils ont appelé ceci «le test du flux d'oxygène » impliquant que des effets microcirculatoires de la prostacycline pouvaient jouer un rôle. Nous avons trouvé particuliérement commode d'utiliser $5 \mu \mathrm{g} \cdot \mathrm{kg}^{-1} \cdot \mathrm{min}^{-1}$ de dobutamine puisqu'à cette dose, la dobutamine augmente sensiblement le débit cardiaque et donc la $\mathrm{DO}_{2}$ mais exerce des effets négligeables sur la pression artérielle. ${ }^{13}$ Une 
TABLEAU Il Interprétation d'un index cardiaque chez le malade grave, en l'absence d'hypozémie ou d'anémic

\begin{tabular}{|c|c|c|c|c|}
\hline Indice cardiaque & $\mathrm{SvO}_{2}$ & Lactate sanguin & Interprétation & Tableau clinique probable \\
\hline$>3.5 \mathrm{~L} \cdot \min ^{-1} \cdot \mathrm{M}^{-2}$ & $>70 \%$ & $<1.5 \mathrm{mEq} \cdot \mathrm{L}^{-1}$ & $\begin{array}{l}\text { extraction d'O } \mathrm{O}_{2} \text { diminuée } \\
\text { pas d'hypoxie tissulaire } \\
\text { débit sanguin élevé (excessif) }\end{array}$ & $\begin{array}{l}\text { surcharge volumique, } \\
\text { fistule } A V \\
\text { agent vasoactif }\end{array}$ \\
\hline$>3.5 \mathrm{~L} \cdot \min ^{-1} \cdot \mathrm{M}^{-2}$ & $>70 \%$ & $>1.5 \mathrm{mEq} \cdot \mathrm{L}^{-1}$ & $\begin{array}{l}\text { extraction d' } \mathrm{O}_{2} \text { diminuée } \\
\text { débit sanguin élevé } \\
\text { hypoxie tissulaire }\end{array}$ & choc septique \\
\hline$>3.5 \mathrm{~L} \cdot \min ^{-1} \cdot \mathrm{M}^{-2}$ & $<65 \%$ & $<1.5 \mathrm{mEq} \cdot \mathrm{L}^{-1}$ & $\begin{array}{l}\dot{\mathrm{V}} \mathrm{O}_{2} \text { élevéc } \\
\text { pas d'hypoxie tissulaire }\end{array}$ & $\begin{array}{l}\text { sepsis, traumatisme, } \\
\text { état postopératoire }\end{array}$ \\
\hline$>3.5 \mathrm{~L} \cdot \min ^{-1} \cdot \mathrm{M}^{-2}$ & $<65 \%$ & $>1.5 \mathrm{mEq} \cdot \mathrm{L}^{-1}$ & $\begin{array}{l}\text { نे } \mathrm{O}_{2} \text { élevée mais } \\
\text { demande en } \mathrm{O}_{2} \text { encore insatisfaite }\end{array}$ & choc septique \\
\hline$<3.0 \mathrm{~L} \cdot \min ^{-1} \cdot \mathrm{M}^{-2}$ & $>70 \%$ & $<1.5 \mathrm{mEq} \cdot \mathrm{L}^{-1}$ & $\begin{array}{l}\text { demande en } \mathrm{O}_{2} \text { abaissée } \\
\text { pas d'hypoxic tissulaire }\end{array}$ & $\begin{array}{l}\text { anesthésie, intoxication } \\
\text { médicamenteuse, hypothermic }\end{array}$ \\
\hline$<3.0 \mathrm{~L} \cdot \min ^{-1} \cdot \mathrm{M}^{-2}$ & $>70 \%$ & $>1.5 \mathrm{mEq} \cdot \mathbf{L}^{-1}$ & $\begin{array}{l}\text { altération d'extraction d' } \mathrm{O}_{2} \\
\dot{\mathrm{V}} \mathrm{O}_{2} \text { basse } \\
\text { (insuffisance cardiaque?) }\end{array}$ & $\begin{array}{l}\text { choc septique avec } \\
\text { hypovolémie ou } \\
\text { dépression myocardique sévère }\end{array}$ \\
\hline$<3.0 \mathrm{~L} \cdot \min ^{-1} \cdot \mathrm{M}^{-2}$ & $<65 \%$ & $<1.5 \mathrm{mEq} \cdot \mathrm{L}^{-1}$ & $\begin{array}{l}\text { débit cardiaque bas } \\
\text { extraction d'O } \mathrm{O}_{2} \text { abaissée } \\
\text { demande en } \mathrm{O}_{2} \text { satisfaite }\end{array}$ & $\begin{array}{l}\text { insuffisance cardiaque, } \\
\text { hypovolémie }\end{array}$ \\
\hline$<3.0 \mathrm{~L} \cdot \min ^{-1} \cdot \mathrm{M}^{-2}$ & $<65 \%$ & $>1.5 \mathrm{mEq} \cdot \mathrm{L}^{-1}$ & $\begin{array}{l}\text { débit cardiaque abaissé } \\
\text { demande en } \mathrm{O}_{2} \text { non satisfaite }\end{array}$ & $\begin{array}{l}\text { choc cardiogénique, hypovolémique } \\
\text { ou obstructif }\end{array}$ \\
\hline
\end{tabular}

dose plus importante de dobutamine pourrait être associée à une augmentation de la $\dot{\mathrm{VO}}_{2}$ chez tous les patients en raison des effets des cathécholamines sur le métabolisme cellulaire.

Le challenge de la $\dot{\mathrm{VO}}_{2}$ suppose que la demande en oxygène du patient reste stable au cours de la procédure si bien que le test ne doit pas prendre plus de 30 minutes et doit être accompli dans un environnement stable et calme. Un soin particulier doit être pris à la mesure précise du débit cardiaque et à la détermination simultanée de la $\mathrm{S}_{\mathrm{v}} \mathrm{O}_{2}$. Le challenge de la $\mathrm{VO}_{2}$ ne nécessite pas de calcul de $\mathrm{DO}_{2}$ et $\dot{\mathrm{VO}}_{2}$ avant et après la procédure car ceci est relativement complexe (et nécessite une calculette). En assumant que les taux d'hémoglobine et de $\mathrm{SaO}_{2}$ ne changent pas au cours du test, l'augmentation du débit cardiaque devrait être compensée par une augmentation significative de la $\mathrm{Sv}_{2}$ si la $\dot{\mathrm{VO}}_{2}$ reste stable. Par contre, une augmentation minimale de la $\mathrm{S}_{\bar{v}} \mathrm{O}_{2}$ indique une augmentation de la $\dot{\mathrm{VO}}_{2}$ associée à l'augmentation de $\mathrm{DO}_{2}$. Chez ces patients, le débit cardiaque est probablement inadéquat par rapport à la demande en oxygène si bien qu'une mesure thérapeutique pourrait être indiquée.

\section{La correction de la dépendance de $\mathrm{V}_{2}$ par rapport à la $\mathrm{DO}_{2}$}

Shoemaker et al..$^{21.22}$ ont indiqué que la survie chez les patients chirurgicaux était associée à des valeurs plus élevées de $\mathrm{VO}_{2}$ et de $\mathrm{DO}_{2}$. De plus, les mesures thérapeutiques visant à maintenir et élever ces valeurs de
$\dot{\mathrm{V}} \mathrm{O}_{2}$ et de $\mathrm{DO}_{2}$ étaient associées à une augmentation de la survie. D'autres investigateurs ${ }^{23,24}$ ont observé que les survivants du SDRA avaient des valeurs plus élevées de $\dot{\mathrm{VO}}_{2}$ et de $\mathrm{DO}_{2}$ que les non-survivants. Ceci pourrait ne pas se retrouver chez des patients présentant un sepsis sévère. Dans une série de 48 patients avec choc septique, nous avons observé $e^{25}$ que les survivants et les nonsurvivants avaient des valeurs similaires de $\dot{\mathrm{VO}}_{2}$ et de $\mathrm{DO}_{2}$. Cependant, les non-survivants avaient des valeurs significativement plus élevées de lactate. Cette observation était interprétée comme un déséquilibre plus profond entre la demande en oxygène et le transport en oxygène chez les patients qui sont décédés. Une élimination plus lente du lactate dans les états de choc plus sévère pourrait également contribuer. Ces éléments représentent un argument contre la considération de valeurs optimales de $\dot{\mathrm{VO}}_{2}$ et de $\dot{\mathrm{VO}}_{2}$ en tant que but thérapeutique au cours du choc septique. Nous pensons que la $\mathrm{VO}_{2}$ est dépendante de la $\mathrm{DO}_{2}$ dans les conditions pathologiques associées à une insuffisance circulatoire aiguë. Lorsque le phénomène de dépendance de la $\dot{V}_{2}$ par rapport à la $\mathrm{DO}_{2}$ est présent, il y a hypoxie tissulaire sous-jacente comme en témoigne le développement de métabolisme anaérobie avec acidose lactique. Dès lors, le phénomène de dépendance de la $\mathrm{VO}_{2}$ par rapport à la $\mathrm{DO}_{2}$ devrait être corrige sans retard puisqu'il représente la base du développement de défaillance multisystémique et finalement de décès. Parfois la demande en oxygène peut être réduite par l'utilisation de ventilation artificielle (pour mettre au 
repos les muscles respiratoires). L'utilisation d'agents inotropes pour compenser la dépression myocardique et augmenter encore la $\mathrm{DO}_{2}$ représente une autre alternative valable. ${ }^{26}$

\section{Références}

1 Cain SM. Appearance of excess lactate in anesthetized dogs during anemic and hypoxic hypoxia. Am J Physiol 1965; 209: 604-8.

2 Fahey JT, Lister G. Postnatal changes in critical cardiac output and oxygen transport in conscious lambs. Am J Physiol 1987; 253: H100-H106.

3 Nelson DP, Beyer C, Samsel RW, Wood LDH, Schumacker $P T$. Pathologic supply/dependency of $\mathrm{O}_{2}$ uptake during bacteremia in dogs. J Appl Physiol 1987; 63: 1487-92.

4 Bihari $D$, Smithies $M$, Gimson A, Tinker $J$. The effects of vasodilatation with prostacyclin on oxygen delivery and uptake in critically ill patients. N Engl J Med 1987; 317 : 397-403.

5 Dantzker $D Z$. Oxygen delivery and utilization in acute and chronic discase. In: Gutierrez G, Vincent JL (Eds.). "Tissue oxygen utilization" - Update in Intensive Care and Emergency Medicine, Vol 12. Heidelberg, Berlin, New York; Springer Verlag 1991; 124-31.

6 Rackow EC, Astiz ME, Weil MH. Cellular oxygen metabolism during sepsis and shock: the relationship of oxygen consumption to oxygen delivery. JAMA $1988 ; 259$ : 1989-93.

7 Geller ER, Jankouskas S, Kirkpatrick J. Mitochondrial death in sepsis: a failed concept. J Surg Res 1986; 40: 514.

8 Haupt MT, Gilbert EM, Carlson RW. Fluid loading increases oxygen consumption in septic patients with lactic acidosis. Am Rev Respir Dis 1985; 131: 912-6.

9. Gilber EM, Haupt MT, Mandanas RY, Huaringa AJ, Carlson $R W$. The effect of fluid loading, blood transfusion, and catecholamine infusion on oxygen delivery and consumption in patients with sepsis. Am Rev Respir Dis 1986; 134: 873-8.

10 Fenwick JC, Dodek PM, Ronco JJ, Phang PT, Wiggs B, Russell $J A$. Increased concentrations of plasma lactate predict pathologic dependence of oxygen consumption on oxygen delivery in patients with adult respiratory distress syndrome. J Crit Care 1990; 5: 81-7.

11 Astiz ME, Rackow EC, Falk JL, Kaufman BS, Weil $M H$. Oxygen delivery and consumption in patients with hyperdynamic septic shock. Crit Care Med 1987; 15: 26-8.

12 Annat G, Viale JP, Percival C, Froment M. Motin $J$. Oxygen delivery and uptake in the adult respiratory distress syndromc: lack of relationship when measured independently in patients with normal blood lactate concentrations. Am Rev Respir Dis 1986: 133: 999-1001.

13 Vincent JL, Roman A, De Backer D, Kahn RJ. $\mathrm{O}_{2}$ supply/dependency: effects of short-term dobutamine infusion. Am Rev Respir Dis 1990; 142: 2-7.

14 Kruse JA, Haupt MT, Puri VK, Carlson RW. Lactate levels as predictors of the relationship between oxygen delivery and consumption in ARDS. Chest 1990; 98: 959-62.

15 Parker MM, Suffredini AF, Natanson C, Ognibene FP, Shelhamer JH, Parrillo JE. Responses of left ventricular function in survivors and nonsurvivors of septic shock. J Crit Care 1989; 4: 19-25.

16 Vincent JL, Reuse C, Frank N, Contempré B, Kahn $R J$. Right ventricular dysfunction in septic shock: assessment by measurement of right ventricular ejection fraction using the thermodilution technique. Acta Anaesthesiol Scand 1989; 33: 34-8.

17 D'Orio $V$, Mendes $P$, Saad $G$, Marcelle $R$. Accuracy in early prediction of prognosis of patients with septic shock by analysis of simple indices: Prospective study. Crit Care Med 1990; 18: 1339-45.

18 Weber KT, Janicki J, Maskin C. Pathophysiology of cardiac failure. Am J Cardiol 1985; 56: 3B-6B.

19 Vincent $J L$. The value of blood lactate monitoring in clinical practice. In: Gutierrez G, Vincent JL (Eds.). "Tissue oxygen utilization" - Update in Intensive Care and Emergency Medicine. Vol 12. Heidelberg, Berlin, New York; Springer Verlag 1991; 260-68.

20 Vincent JL, Dufaye P, Berré J, Leeman M, Degaute JP. $K a h n R J$. Serial lactate determinations during circulatory shock. Crit Care Med 1983; 11 : 449-51.

21 Shoemaker WC, Appel PL, Kram HB, Waxman K, Lee $T$. Prospective trial of supranormal values of survivors as therapeutic goals in high-risk surgical patients. Chest 1988; 94: 1176-86.

22 Shoemaker WC, Kram HB, Appel PL, Fleming AW. The efficacy of central venous and pulmonary artery catheters and therapy based upon them in reducing mortality and morbidity. Arch Surg 1990; 125: 1332-8.

23 Russell JA, Ronco JJ, Lockhat D, Belzberg A, Kiess $M$. Dodek $P M$. Oxygen delivery and consumption and ventricular preload are greater in survivors than in nonsurvivors of the adult respiratory distress syndrome. Am Rev Respir Dis 1990; 141: 659-65.

24 Cryer HG, Richardson JD, Longmire-Cook S, Brown $M$. Oxygen delivery in patients with adult respiratory distress syndrome who undergo surgery. Arch Surg 1989; 124: 1378-85.

25 Bakker J, Vincent $J L$. Blood lactate levels are superior to oxygen derived variables in predicting outcome in human shock. Chest (in press).

26 Vincent JL, Roman A, Kahn RJ. Dobutamine administration in septic shock: addition to a standard protocol. Crit Care Med 1990; 18: 689-93. 


\section{Oxygen delivery in the critically ill}

The relationship between oxygen uptake $\left(\mathrm{VO}_{2}\right)$ and oxygen supply $\left(\mathrm{DO}_{2}\right)$ has been a subject of increasing interest in intensive care medicine. $\mathrm{DO}_{2}$ is the product of cardiac output and arterial oxygen content $\left(\mathrm{CaO}_{2}\right)$. $\mathrm{CaO}_{2}$ is in turn determined by the haemoglobin concentration and saturation. A low haemoglobin level should be corrected by blood transfusion whenever it threatens $\mathrm{DO}_{2}$. Likewise, a low arterial oxygen pressure $\left(\mathrm{PaO}_{2}\right)$ should be corrected in any hypoxaemic patient. The interpretation of a cardiac output value is more complex since cardiac output adapts to the $\mathrm{O}_{2}$ needs of the body. A low cardiac output should be treated only when $\mathrm{O}_{2}$ demand is normal or high. In contrast a low cardiac output can still be adequate in an anaesthetized, hypothermic, mechanically ventilated patient. At the other extreme, since oxygen demand of the tissues is often increased in acute diseases associated with sepsis, surgery, trauma, or other inflammatory states, cardiac output must sometimes increase markedly in these circumstances. To some extent, these conditions can be compared to exercice.

When oxygen demand increases acutely, $\mathrm{DO}_{2}$ must also readily increase. An increase in $\mathrm{PaO}_{2}$ above normal does not increase $\mathrm{DO}_{2}$ much because of the nearly complete oxygen saturation of the haemoglobin. Similarly, an increase in haemoglobin concentration above normal cannot be achieved rapidly, and would not efficiently increase cellular $\mathrm{O}_{2}$ availability because blood viscosity would rise simultaneously. Hence, cardiac output and blood flow distribution in the organism represent the only factors that can readily and efficiently increase cellular $\mathrm{O}_{2}$ availability. Accordingly, a cardiac output value that is considered normal might still be inadequate or insufficient when tissue $\mathrm{O}_{2}$ demand is elevated.

Study of the relationship between $\dot{\mathrm{V}}_{2}, \mathrm{DO}_{2}$ and oxygen demand helps to interpret cardiac output values. It also allows the development of an unique concept defining all types of acute circulatory failure and can indicate therapeutic options that are available to improve cellular function and prevent the development of multiple organ failure and death.

The $\mathrm{VO}_{2} / \mathrm{DO}_{2}$ relationship in physiological conditions In stable physiological conditions, $\dot{\mathrm{VO}}_{2}$ is equal to oxygen demand. In the absence of tissue hypoxia, blood lactate concentrations are normal at about $1 \mathrm{mmol} \cdot \mathrm{L}^{-1}$. If

\section{J.L. Vincent}

$\mathrm{DO}_{2}$ decreases in the presence of hypoxaemia, anaemia or low cardiac output, $\mathrm{VO}_{2}$ remains relatively stable over a wide range of $\mathrm{DO}_{2}$ values. Indeed, the $\mathrm{O}_{2}$ extraction, i.e., the ratio of the arteriovenous $\mathrm{O}_{2}$ difference to $\mathrm{CaO}_{2}$, or the $\mathrm{VO}_{2} / \mathrm{DO}_{2}$ ratio, increases. The primary underlying mechanism lies in the microvasculature, where the capillary network dilates to provide more $\mathrm{O}_{2}$ to the cells. $\mathrm{VO}_{2}$ is therefore $\mathrm{DO}_{2}$ - or supply-independent. Of course, this is true only when the $\mathrm{O}_{2}$ demand is stable. Should the $\mathrm{O}_{2}$ demand increase for any reason (during exercise, for example), the cardiac output and therefore the $\mathrm{DO}_{2}$ would increase simultaneously.

When $\mathrm{DO}_{2}$ decreases below a critical value, the increase in $\mathrm{O}_{2}$ extraction becomes less efficient, so that the $\mathrm{VO}_{2}$ starts to decrease. The $\mathrm{VO}_{2}$ then becomes $\mathrm{DO}_{2}$ or supply-dependent. In the intact animal, this critical level is about 8 to $10 \mathrm{ml} \cdot \mathrm{kg}^{-1} \cdot \mathrm{min}^{-1} \cdot{ }^{1-3}$ As shown by Cain ${ }^{1}$ more than $25 \mathrm{yr}$ ago, these states are associated with tissue hypoxia, as reflected by increased blood lactate concentrations. In conscious lambs, Fahey and Lister ${ }^{2}$ observed that $\mathrm{VO}_{2}$ became $\mathrm{DO}_{2}$-dependent when cardiac output was reduced below a critical level. Lactic acidosis also developed when this critical level of cardiac output was reached. Hence, this $\mathrm{VO}_{2} / \mathrm{DO}_{2}$ dependency characterizes the types of circulatory shock associated with a profound reduction in cardiac output: the hypovolaemic, cardiogenic and obstructive types of shock. In pure cardiogenic or obstructive types of shock, the $\mathrm{O}_{2}$ extraction capabilities of the organism are very efficient, so that lactate levels start to rise only in extreme situations, which are associated with high mortality. In hypovolaemic shock, a combination of a low cardiac output (by a reduction in venous retum) and a diminished blood volume accounts for the tissue hypoxia.

\section{The $\mathrm{VO}_{2} / \mathrm{DO}_{2}$ dependency in sepsis}

In patients with sepsis or with the adult respiratory distress syndrome (ARDS), $\mathrm{VO}_{2}$ can remain dependent on $\mathrm{DO}_{2}$ even when the $\mathrm{DO}_{2}$ is normal or high. ${ }^{4.5}$ These states are typically associated with a high $\mathrm{O}_{2}$ demand, so that cardiac output and $\mathrm{DO}_{2}$ are expected to be high. At the same time, $\mathrm{O}_{2}$ extraction by the tissues is altered in these inflammatory states by several factors, including the development of microthrombi, the release of various vasoactive mediators, and the alteration of endothelial 
cell function. ${ }^{6}$ Cellular alterations in sepsis appear to develop later than these microvascular changes. ${ }^{7}$

Several pieces of evidence indicate that the pathological $\dot{\mathrm{VO}}_{2} / \mathrm{DO}_{2}$ dependency, like the physiological $\dot{\mathrm{VO}}_{2} /$ $\mathrm{DO}_{2}$ dependency, is associated with tissue hypoxia and the development of anaerobic metabolism. However, the alterations in oxygen extraction make the organism more susceptible to an inappropriate $\mathrm{DO}_{2}$. Experimentally, Nelson et al. ${ }^{3}$ observed during graded haemorrhage in dogs that the critical $\mathrm{DO}_{2}$ level, below which the $\mathrm{VO}_{2} / \mathrm{DO}_{2}$ dependency occurred, was associated with the development of increased blood lactate levels. These observations were in agreement with the results of the Cain ${ }^{1}$ studies in which $\mathrm{DO}_{2}$ was progressively lowered by anaemia or hypoxaemia. When a similar protocol was applied after administration of endotoxin or live bacteria to the animal, the same course of events was observed, except that the critical $\mathrm{DO}_{2}$ was displaced toward higher values. ${ }^{3}$ Hence, a reduced $\mathrm{DO}_{2}$ level that was still well tolerated in the intact animal could be associated with tissue hypoxia and lactic acidosis in the septic animal.

In patients also, the $\mathrm{VO}_{2} / \mathrm{DO}_{2}$ dependency is usually associated with lactic acidosis. In patients with sepsis and lactic acidosis, several investigators ${ }^{8-11}$ observed an increase in $\mathrm{VO}_{2}$ associated with an increase in cardiac output after fluid challenge. Such an increase was not observed in patients without lactic acidosis. ${ }^{8-10}$ Fenwick et al. ${ }^{10}$ observed in patients with ARDS that blood transfusion increased $\mathrm{VO}_{2}$ only in those with elevated lactate levels. In contrast, Annat et al. ${ }^{12}$ did not observe the $\mathrm{VO}_{2} / \mathrm{DO}_{2}$ dependency in patients with normal blood lactate levels.

We studied ${ }^{13}$ the $\mathrm{DO}_{2}$ and $\mathrm{VO}_{2}$ responses to the administration of a standard dose of $5 \mu \mathrm{g} \cdot \mathrm{kg}^{-1} \cdot \mathrm{min}^{-1}$ of dobutamine in 78 critically ill patients with heart failure or sepsis. In both subsets of patients, $\mathrm{VO}_{2}$ increased only in those with elevated blood lactate concentrations. Among ARDS patients in the septic group, $\mathrm{VO}_{2}$ increased only when lactic acidosis was present. ${ }^{13}$

The results of these studies and others ${ }^{14}$ demonstrate that the $\mathrm{VO}_{2} / \mathrm{DO}_{2}$ dependency is observed only in the presence of tissue hypoxia as reflected by increased blood lactate levels. Thus the increase in $\mathrm{VO}_{2}$ achieved in these conditions is probably beneficial. This is important, since another interpretation is that the increased amount of $\mathrm{O}_{2}$ being consumed is primarily utilized for non-ATPproducing systems related to the inflammatory processes. This use of $\mathrm{O}_{2}$ could contribute to the formation of free oxygen radicals and could thus be detrimental to the organism. If these mechanisms did occur, they would also be observed in the absence of tissue hypoxia and lactic acidosis. In the presence of tissue hypoxia, however, the additional $\mathrm{O}_{2}$ is preferentially used in the mitochondria,

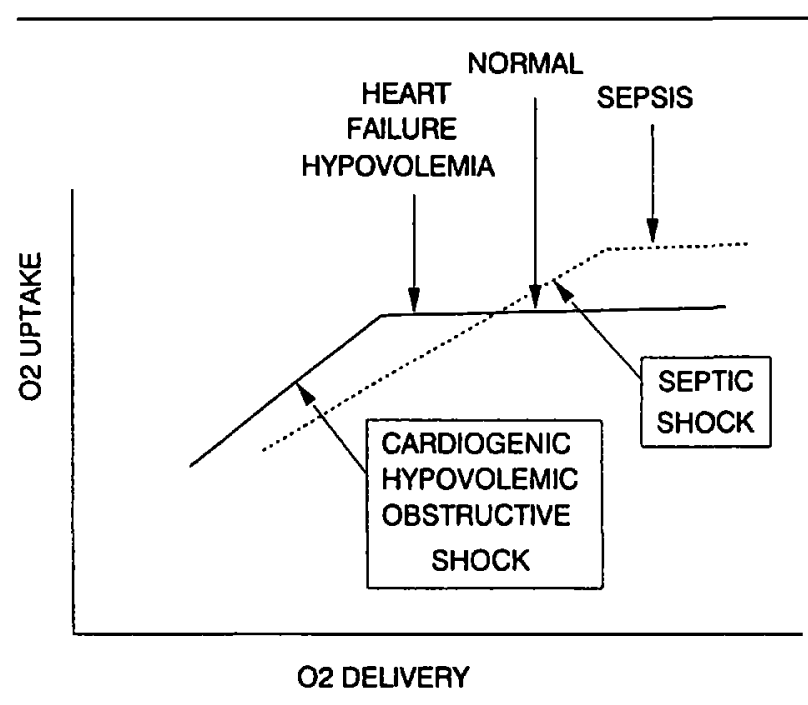

FIGURE Relationship between oxygen uptake and delivery in normal patients and in heart failure, hypovolaemia, sepsis and shock.

as the affinity of the extramitochondrial oxidase systems is lower than the mitochondrial $\mathrm{O}_{2}$ transport chain (cytochrome aa3). Extramitochondrial $\mathrm{O}_{2}$ utilization might also take place, but in late stages of sepsis when multiple organ failure has already occurred.

The insufficient $\mathrm{DO}_{2}$ in the presence of some acute diseases characterized by an elevated $\mathrm{O}_{2}$ demand and an altered oxygen extraction can be explained by the myocardial depression that is associated with severe sepsis. A decreased myocardial contractility can be documented even when cardiac output is normal or elevated. ${ }^{15-17}$

These elements can account for the abnormalities associated with all forms of acute circulatory failure (shock). Hypovolaemic, cardiogenic and obstructive types of shock are characterized by a reduction in $\mathrm{DO}_{2}$, but oxygen demand is relatively normal and oxygen extraction is well preserved (Figure). On the other hand, septic shock is characterized by an increase in oxygen demand, an alteration in oxygen extraction and a limitation in cardiac output by the underlying (sometimes unsuspected) myocardial depression. The degree of myocardial depression seems directly related to the severity of sepsis and the likelihood of death. ${ }^{16,17}$

\section{Interpretation of a cardiac output value}

In view of these findings, we suggest five successive steps in the interpretation of cardiac output (Table I).

The first step is to relate cardiac output to the size of the patient. Reference to the body surface area is made to obtain a cardiac index value.

The second step is to determine the presence of anaemia or hypoxaemia, and, if $\mathrm{DO}_{2}$ can be threatened, this should be corrected. A haemoglobin concentration of 10 to 
TABLE I Steps in the interpretation of a cardiac output value in a critically ill patient

I Refer to the body surface area (calculatc cardiac index)

2 Rule out hypoxaemia or anaemia (and correct them if present)

3 Determine $\mathrm{Sv}_{2}$

4 Determine blood lactate concentration

5 Challenge the $\mathrm{VO}_{2}$

$11.5 \mathrm{~g} \cdot \mathrm{dl}^{-1}$ (or a haematocrit of 30 to $35 \%$ ) is usually

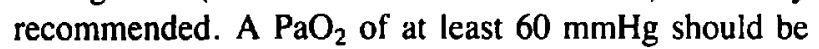
maintained to obtain a haemoglobin saturation of at least 90\%.

The third step consists of the simultaneous measurement of mixed venous $\mathrm{O}_{2}$ saturation $\left(\mathrm{S}_{\bar{v}} \mathrm{O}_{2}\right)$. Changes in $\mathrm{S} \overline{\mathrm{V}} \mathrm{O}_{2}$ accompany changes in $\mathrm{VO}_{2} / \mathrm{DO}_{2}$ : at a given $\dot{\mathrm{V}}_{2}$, a decrease in cardiac output is associated with a concurrent decrease in $\mathrm{S}_{\bar{v}}{ }_{2}$. The interpretation of $\mathrm{S}_{\bar{v}} \mathrm{O}_{2}$ is, however, limited by the possibility of altered $\mathrm{O}_{2}$ extraction capabilities by the tissues. The critical extraction point can be defined in precise laboratory investigations but not clinically.

The fourth step consists of the measurement of blood lactate concentration. $\mathrm{Sv}_{2}$ is no substitute for blood lactate and vice versa, because the two are not expected to correlate well except perhaps in the presence of isolated severe heart failure. ${ }^{18}$ Lactate levels reflect the degree of anaerobic metabolism. Their interpretation can be com- plicated by the fact that they can also increase in other conditions, including seizures, decompensated diabetes or some intoxications. Of more importance, blood lactate levels are influenced not only by lactate production but also by lactate elimination. As lactate is metabolized predominantly by the liver, it can remain elevated when hepatic function is altered. Nevertheless, lactate levels are normal in stable patients with advanced liver insufficiency, so that normal blood lactate levels remain a strong argument to refute the presence of tissue hypoxia. ${ }^{19}$ This reduced lactate clearance can complicate the interpretation of its blood levels during the evolution of an episode of acute circulatory failure. Therefore, it is important to measure lactate levels serially so as to allow a dynamic interpretation of the values. In the best circumstances, associated with a good response to fluid therapy, lactate levels decrease by at least $10 \%$ during the first hour of treatment. ${ }^{20}$ Table II shows how cardiac output is interpreted according to $\mathrm{S}_{\mathrm{v}} \mathrm{O}^{12}$ and lactate levels.

The fifth step consists of a dynamic evaluation of $\mathrm{VO}_{2}$ before and after a transient increase in cardiac output. This " $\mathrm{VO}_{2}$ challenge" can be accomplished with fluid administration or blood transfusion. Bihari et al. ${ }^{4}$ showed that a dose of $5 \mathrm{ng} \cdot \mathrm{kg}^{-1} \cdot \mathrm{min}^{-1}$ of prostacyclin could reveal the $\dot{\mathrm{VO}} \mathrm{O}_{2} / \mathrm{DO}_{2}$ dependency in patients with sepsis or acute respiratory failure; they called this the " $\mathrm{O}_{2}$ flux test." implying that microcirculatory effects of prostacy-

TABLE II Interpretation of a cardiac index value in the critically ill patient, in the absence of hypoxaemia or anaemia

\begin{tabular}{|c|c|c|c|c|}
\hline Cardiac index & $\mathrm{S} \tilde{\mathrm{v}} \mathrm{O}_{2}$ & Blood lactate & Interpretation & Probable setting \\
\hline$>3.5 \mathrm{~L} \cdot \min ^{-1} \cdot \mathrm{M}^{-2}$ & $>70 \%$ & $<1.5 \mathrm{mEq} \cdot \mathrm{L}^{-1}$ & $\begin{array}{l}\text { Reduced } \mathrm{O}_{2} \text { extraction } \\
\text { No tissue hypoxia } \\
\text { High (excessive) blood flow }\end{array}$ & $\begin{array}{l}\text { Fluid overload, AV fistula } \\
\text { vasoactive agent }\end{array}$ \\
\hline$>3.5 \mathrm{~L} \cdot \mathrm{min}^{-1} \cdot \mathrm{M}^{-2}$ & $>70 \%$ & $>1.5 \mathrm{mEq} \cdot \mathrm{L}^{-1}$ & $\begin{array}{l}\text { Reduced } \mathrm{O}_{2} \text { extraction } \\
\text { High blood flow } \\
\text { Tissue hypoxia }\end{array}$ & Septic shock \\
\hline$>3.5 \mathrm{~L} \cdot \min ^{-1} \cdot \mathrm{M}^{-2}$ & $<65 \%$ & $<1.5 \mathrm{mEq} \cdot \mathrm{L}^{-1}$ & $\begin{array}{l}\text { Elevated } \mathrm{VO}_{2} \\
\text { No tissue hypoxia }\end{array}$ & $\begin{array}{l}\text { Sepsis, trauma, } \\
\text { postsurgery }\end{array}$ \\
\hline$>3.5 \mathrm{~L} \cdot \min ^{-1} \cdot \mathrm{M}^{-2}$ & $<65 \%$ & $>1.5 \mathrm{mEq} \cdot \mathrm{L}^{-1}$ & $\begin{array}{l}\text { Elevated } \mathrm{VO}_{2} \text { but } \\
\mathrm{O}_{2} \text { demand still unsatisfied }\end{array}$ & Septic shock \\
\hline$<3.0 \mathrm{~L} \cdot \min ^{-1} \cdot \mathrm{M}^{-2}$ & $>70 \%$ & $<1.5 \mathrm{mEq} \cdot \mathrm{L}^{-1}$ & $\begin{array}{l}\text { Low } \mathrm{O}_{2} \text { demand } \\
\text { No tissue hypoxia }\end{array}$ & $\begin{array}{l}\text { Anaesthesia, drug over- } \\
\text { dose, hypothermia }\end{array}$ \\
\hline$<3.0 \mathrm{~L} \cdot \min ^{-1} \cdot \mathrm{M}^{-2}$ & $>70 \%$ & $>1.5 \mathrm{mEq} \cdot \mathrm{L}^{-1}$ & $\begin{array}{l}\text { Altered } \mathrm{O}_{2} \text { extraction } \\
\text { Low } \mathrm{VO}_{2} \\
\text { (Myocardial failurc?) }\end{array}$ & $\begin{array}{l}\text { Septic shock with } \\
\text { hypovolaemia or severe } \\
\text { myocardial depression }\end{array}$ \\
\hline$<3.0 \mathrm{~L} \cdot \min ^{-1} \cdot \mathrm{M}^{-2}$ & $<65 \%$ & $<1.5 \mathrm{mEq} \cdot \mathrm{L}^{-1}$ & $\begin{array}{l}\text { Low cardiac output } \\
\text { Increased } \mathrm{O}_{2} \text { extraction } \\
\mathrm{O}_{2} \text { demand satisfied }\end{array}$ & $\begin{array}{l}\text { Hean failure } \\
\text { hypovolacmia }\end{array}$ \\
\hline$<3.0 \mathrm{~L} \cdot \min ^{-1} \cdot \mathrm{M}^{-2}$ & $<65 \%$ & $>1.5 \mathrm{mEq} \cdot \mathrm{L}^{-1}$ & $\begin{array}{l}\text { Low cardiac output } \\
\mathrm{O}_{2} \text { demand unsatisfied }\end{array}$ & $\begin{array}{l}\text { Cardiogenic, hypovo- } \\
\text { lacmic, obstructivc shock }\end{array}$ \\
\hline
\end{tabular}


clin could play a role. We have found it particularly convenient to give $5 \mu \mathrm{g} \cdot \mathrm{kg}^{-1} \cdot \mathrm{min}^{-1}$ dobutamine because at this dose dobutamine has significant effects on cardiac output and thus $\mathrm{DO}_{2}$, but minimal effects on blood pressure. ${ }^{13} \mathrm{~A}$ larger dose of dobutamine could be associated with an increase in $\mathrm{VO}_{2}$ in all patients because of the effects of catecholamines on cellular metabolism.

The $\mathrm{VO}_{2}$ challenge requires that the $\mathrm{O}_{2}$ demand of the patient remains stable during the procedure, and thus the test should not last more than $30 \mathrm{~min}$ and should be performed in a stable, quiet environment. Particular care must be taken to measure cardiac output accurately and to perform the $\mathrm{S}_{\overline{\mathrm{O}}} \mathrm{O}_{2}$ measurement simultaneously. The $\mathrm{VO}_{2}$ challenge does not require calculation of $\mathrm{DO}_{2}$ and $\mathrm{VO}_{2}$ before and after the procedure, as this is cumbersome (and requires a pocket calculator). Assuming that haemoglobin and $\mathrm{SaO}_{2}$ levels do not change during the test, the increase in cardiac output should be matched by a significant increase in $\mathrm{SvO}_{2}$ if $\dot{\mathrm{VO}}_{2}$ remains stable. In contrast, a minimal increase in $\mathrm{S}_{\bar{v}} \mathrm{O}_{2}$ indicates an increase in $\dot{\mathrm{VO}}_{2}$ associated with the increase in $\mathrm{DO}_{2}$. In this type of patient, cardiac output is probably not adequate to meet the $\mathrm{O}_{2}$ demand, so that a therapeutic measure could be indicated.

The correction of the $\mathrm{VO}_{2} / \mathrm{DO}_{2}$ dependency

Shoemaker et al. ${ }^{21,22}$ indicated that survival in surgical patients was associated with higher $\mathrm{VO}_{2}$ and $\mathrm{DO}_{2}$ values. Moreover, therapeutic measures aiming at maintaining high $\dot{\mathrm{VO}}_{2}$ and $\mathrm{DO}_{2}$ values were associated with increased survival. Other investigators ${ }^{23,24}$ observed that survivors from ARDS had higher $\dot{\mathrm{V}}_{2}$ and $\mathrm{DO}_{2}$ values than nonsurvivors. This might not be true in patients with severe sepsis. In a series of 48 patients with septic shock, we observed $^{25}$ that survivors and non-survivors had similar $\dot{\mathrm{VO}} \mathrm{O}_{2}$ and $\mathrm{DO}_{2}$ values. However, non-survivors had higher blood lactate levels. This observation was interpreted as a more profound imbalance between oxygen demand and oxygen transport in patients who died. A greater impairment in lactate clearance in more severe shock conditions could also contribute. These data argue against the consideration of optimal $\dot{\mathrm{VO}}_{2}$ and $\mathrm{DO}_{2}$ values as therapeutic goals in septic shock. We believe that the $\dot{\mathrm{V}} \mathrm{O}_{2}$ is dependent on $\mathrm{DO}_{2}$ in pathological conditions associated with acute circulatory failure. When $\mathrm{VO}_{2} / \mathrm{DO}_{2}$ dependency is present, there is underlying tissue hypoxia, as reflected by the development of anaerobic metabolism and lactic acidosis. Thus $\mathrm{VO}_{2} / \mathrm{DO}_{2}$ dependency should be corrected without delay, as it sets the stage for multiple organ failure and ultimate death. Sometimes, oxygen demand can be reduced by the use of mechanical ventilation (to put the respiratory muscles at rest). The use of inotropic agents to compensate for the myocardial depression and further increase $\mathrm{DO}_{2}$ represents another valuable alternative. ${ }^{26}$

\section{References \\ (See page R43)}

